\title{
Messung der Startphotonenzahl eines Neodym-Laseroszillators
}

von $B$. HAUSHERR und H. WEBER

(Institut für angewandte Physik der Universität Bern)

Beim aktiven $Q$-Switch-Laser besteht eine zeitliche Verzögerung $\tau$ zwischen Öffnen des optischen Schalters und Einsetzen der Sättigung. Diese Zeit $\tau$ und damit auch die spektrale Einengung des durch die Spontanemission (Startphotonen) ausgelösten Lasersignals sind abhängig von der Durchgangsverstärkung und der Startphotonenzahl. Die Kenntnis dieser Grössen ist u.a. notwendig für die optimale Einstellung der Parameter beim passiven mode-locking-Betrieb [1]. An einem sehr stabilen Nd-Glas und Nd-YAG-Laser mit $\mathrm{TEM}_{00}$-Emission wurden Verzögerungszeit $\tau$ und Durchgangsverstärkung gemessen und daraus die Startphotonenzahl bestimmt. Sie ergab sich übereinstimmend mit der Theorie [2] im Mittel zu einigen Photonen pro Mode im Resonator.

Eine ausführliche Arbeit erscheint demnächst in der ZAMP.

\section{Literatur}

[1] N. G. BASOV et al., IEEE J. QE-4, 606 (1968).

[2] A. Van der Ziel, Proc. IEEE 52, 1738 (1964).

\section{Pumpkavität für einen Festkörperlaser mit idealer Strahlgeometrie}

von H. U. Leuenberger und J. StefFen

(Institut für angewandte Physik der Universität Bern)

Die Mehrzahl der heute gebräuchlichen Lasersysteme ist ausgelegt für hohe Ausgangsleistung, wobei die Strahlqualität eine untergeordnete Rolle spielt. Mit zunehmender Verwendung von Lasern für spezielle Untersuchungen (z.B. Streuexperimente, Materialbearbeitung) wird die räumliche und zeitliche Struktur der Laserstrahlung von Bedeutung. Um die gesteigerten Anforderungen an die Strahlqualität zu erfüllen, wird in den meisten Fällen Grundmodestrahlung hoher Intensität erforderlich sein.

Die Erzeugung intensiver und reproduzierbarer Grundmodestrahlung mit Festkörperlasern ist aus mehreren Gründen erschwert. Erstens entsteht durch Energieübertragung beim optischen Pumpen im Laserstabquerschnitt ein radialer Temperaturgradient; der Stab wirkt als thermische Linse und schränkt die Ausdehnung des Grundmodes ein. Bei rotationssymmetrischer Ausleuchtung kann die Linsenwirkung durch geeignete Wahl des Laserresonators kompensiert werden [1]. Zweitens wird bei unsymmetrischer Pumplichtverteilung im Stabquerschnitt die Laserstabachse verkrümmt und dadurch die Resonatorgeometrie gestört. Drittens ist bei kommerziellen Geräten der Laserstab oft mechanisch nicht genügend stabil gehaltert, was die Erzeugung reproduzierbarer Grundmodestrahlung im Dauerbetrieb ausschliesst.

Zur Erzeugung von intensiver und reproduzierbarer Grundmodestrahlung muss demnach der Laserstab möglichst homogen ausgeleuchtet sein und mechanisch stabil gehaltert werden. 